\title{
Reviving fossil radio plasma in clusters of galaxies by adiabatic compression in environmental shock waves
}

\author{
T. A. Enßlin ${ }^{1}$ and Gopal-Krishna ${ }^{2}$ \\ 1 Max-Planck-Institut für Astrophysik, Karl-Schwarzschild-Str.1, 85741 Garching, Germany \\ 2 National Centre for Radio Astrophysics, Tata Institute of Fundamental Research, Pune University Campus, \\ Ganeshkhind, Pune 411007, India
}

Received 30 March 2000 / Accepted 3 November 2000

\begin{abstract}
We give for a plasma with a history of several expansion and contraction phases an analytical model of the evolution of a contained relativistic electron population under synchrotron, inverse Compton and adiabatic energy losses or gains. This is applied to different scenarios for evolution of radio plasma inside the cocoons of radio galaxies, after the activity of the central engine has ceased. It is demonstrated that fossil radio plasma with an age of even up to 2 Gyr can be revived by compression in a shock wave of large-scale structure formation, caused during the merging events of galaxy clusters, or by the accretion onto galaxy clusters. We argue, that this is a highly plausible explanation for the observed cluster radio relics, which are the regions of diffuse radio emission found in clusters of galaxies, without any likely parent radio galaxy seen nearby. An implication of this model is the existence of a population of diffuse, ultra-steep spectrum, very low frequency radio sources located inside and possibly outside of clusters of galaxies, tracing the revival of aged fossil radio plasma by the shock waves associated with large-scale structure formation.
\end{abstract}

Key words. acceleration of particles - shock waves - galaxies: intergalactic medium - galaxies: active - galaxies: clusters: general - radio continuum: general

\section{Introduction}

The radio cocoons of radio galaxies become rapidly undetectable after the central engine of the active galactic nucleus ceases to inject fresh radio plasma, or even earlier (Kaiser et al. 2000, and references therein). Radio plasma consists of relativistic electrons and magnetic fields, which are responsible for the synchrotron radio emission, and possibly relativistic protons and/or a non-relativistic thermal gas component.

Although undetectable, aged radio plasma should still be an important component of the inter-galactic medium (IGM). We investigate the possibility of reviving patches of such fossil radio plasma (also called radio ghosts, Enßlin 1999a) by compression in a shock wave produced in the IGM by the flows of cosmological large-scale structure formation. Such shock waves re-energize the electron population in the fossil radio cocoons, which can lead to observable synchrotron emission. This is probably the mechanism responsible for the, so called, cluster radio relics, which are patches of diffuse, sometimes polarized radio emission typically found at peripheral locations in clusters of galaxies. These cluster radio relics cannot be simply relic radio galaxies, as their name suggests. The spectral ages of the electron population are usually too

Send offprint requests to: T. A. Enßlin e-mail: ensslin@mpa-garching.mpg.de short to admit even the nearest galaxy to have been the parent radio galaxy, which has moved to its present location with a velocity typical for cluster galaxies. Therefore, a recent enhancement of the nonthermal radio output of the cluster relic sources is mandatory.

For reviews on diffuse cluster radio emission (cluster radio relic and halos, which are believed to be distinct phenomena), see Jaffe (1992), Feretti \& Giovannini (1996), Enßlin et al. (1998), Feretti (1999), Enßlin (1999b), and Giovannini et al. (1999).

The connection between the presence of a shock wave and the appearance of the cluster radio relic phenomena was assumed to be due to Fermi-I shock acceleration of electrons, in Enßlin et al. (1998) and Roettiger et al. (1999). While this process might work within the normal IGM, several arguments favor the possibility that indeed old fossil radio plasma is revived in the case of cluster relic sources:

- Cluster radio relics are extremely rare, whereas shock waves should be very common within clusters of galaxies. The dual requirement of a shock wave and fossil radio plasma, for producing a cluster radio relic, would be an attractive explanation for the rareness of the relics;

- Fossil radio plasma with existing relativistic electron population and fairly strong magnetic field appears to 
have ideal properties to be brightened up during the shock's passage;

- The cluster radio relic $1253+375$ near the Coma cluster of galaxies appears to be fed with radio plasma by the nearby galaxy NGC 4789 (see Fig. 1 and scenario C in Sect. 6).

But, if indeed the fossil radio plasma and not the normal IGM were to become radio luminous at a shock wave, the expected very high sound velocity of that relativistic plasma should forbid the shock in the ambient medium to penetrate into the radio plasma. Thus, shock acceleration is not expected to occur there. Instead, the fossil radio plasma would get adiabatically compressed, and the energy gain of the electrons is expected to be mainly due to adiabatic heating. It is the purpose of this work to demonstrate that this process is sufficient to account for the cluster radio relics.

Sections 2 and 3 discuss the expected properties of the components of this model: shock waves and fossil radio plasma. In Sect. 4 we give a mathematical formalism to describe the evolution of the radiation spectrum during the history of a radio cocoon. The different phases of this history are described in Sect. 5 and applied to three different scenarios in Sect. 6. The discussion of the merits and potential deficiencies of this model can be found in Sect. 7 .

\section{Structure formation shock waves}

The flows of the cosmological large-scale structure formation are predicted to produce frequently shock waves at the boundaries of clusters and filaments of galaxies and during cluster merger (Quilis et al. 1998; Miniati et al. 2000b). But only in a few cases cluster merger shock waves could be detected directly as temperature structures in the X-ray emitting cluster gas. The best examples are in Abell 2256 (Briel \& Henry 1994) and in Abell 1367 (Donnelly et al. 1998). The reason for the small X-ray detectability of shock waves is the necessary simultaneous spatial and spectral resolution of the instrument, combined with a sufficiently large collecting area to yield adequate photon statistics from the often peripheral shock waves in clusters. For instance one of the best studied cluster, the Coma cluster, is proposed to contain a shock wave at the location of the diffuse radio source $1253+275$ (see Fig. 1; Enßlin et al. 1998; this work). The available X-ray map (White et al. 1993) shows an extension of the $\mathrm{X}$-ray emitting gas in the direction of this radio source, but the sensitivity is too low to reveal any structure at its location. The feasibility of shock wave detections with new instruments as the XMM experiment is estimated in Tozzi et al. (2000). It is interesting to note that at the locations of the two above mentioned X-ray detections of shock waves, cluster radio relics are observed (Enßlin et al. 1998). This indicates that these sources are indeed tracers of shock waves. The importance of a detailed understanding of these tracers of shock waves is underscored by the shock wave's possible roles in intergalactic magnetic field

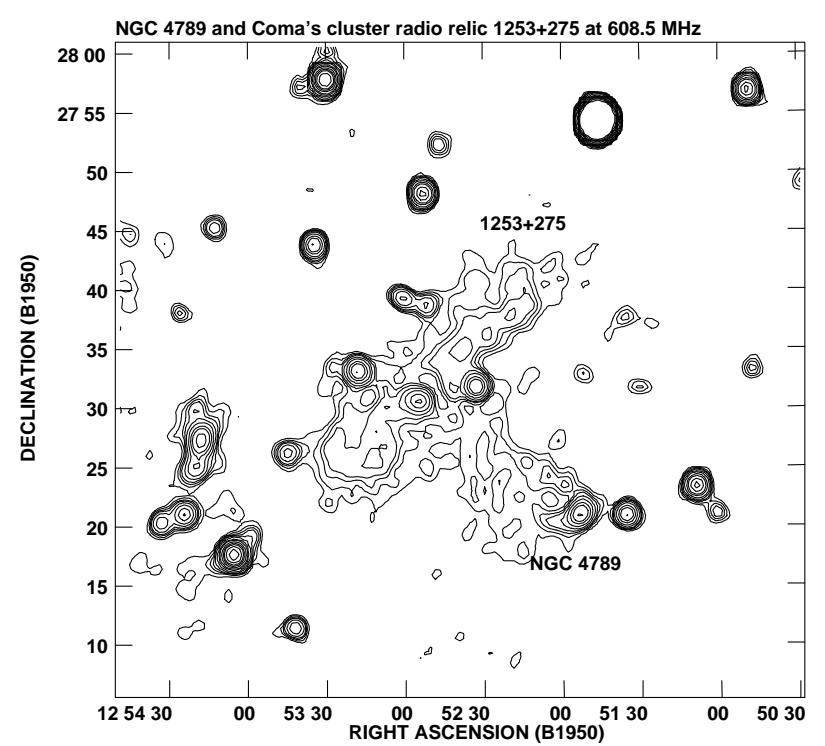

Fig. 1. Radio map of the cluster radio relic $1253+275$ in the Coma cluster from Giovannini et al. (1991) and its probable source of radio plasma: the narrow angle tailed radio galaxy NGC 4789. The radio tails of NGC 4789 indicate the direction from which this galaxy came: the cluster center which is located outside this map. A radio polarization of $27 \%$ was detected from the radio relic (Giovannini et al. 1991), but not from the tails of NGC 4789 feeding the relic with radio plasma. This is a clear signature of the alignment of magnetic fields enhanced in the shock compression. The polarization vectors are consistent with a shock wave oriented parallel to the main axis of the radio relic (Enßlin et al. 1998)

generation (Kulsrud et al. 1997; Ryu et al. 1998), cosmic ray acceleration (Norman et al. 1995; Kang et al. 1996; Kang et al. 1997; Siemieniec \& Ostrowski 2000; Miniati et al. 2000a), gamma ray background production (Loeb \& Waxman 2000), and in probing the entropic history of the IGM (Tozzi et al. 2000).

\section{Fossil radio plasma}

In this work it is assumed that the radio plasma in the lobes of radio galaxies remains mostly intact after release, forming the proposed radio fossils or ghosts. This is specified and discussed in the following. Important for the presented model is that the magnetic fields and ultrarelativistic electron population continue to remain within the lobe for up to 2 Gyr. Transport or decay processes of magnetic fields, and relativistic particles populations need to be slower than this time scale.

Clearly, the light radio plasma is subject to buoyancyinduced motions in the cluster gravitational potential. The action of buoyancy is e.g. able to explain the morphology of the eastern radio lobe of M 87 (Churazov et al. 2000). But since this does not separate the electrons and fields, the fossils would not get disintegrated. Nevertheless, relative motion at a plasma-field interface (e.g. the surface of a buoyant fossil) can produce enhanced mixing (Lerche \& Parker 1967) due to finite gyro-radius effects 
of the ions in the embedding gas. This should produce a mixing layer of the order of several gyro-radii of the ions $\left(10^{-13} \ldots 10^{-11} \mathrm{kpc}\right)$. But this would soften the magnetic boundary and therefore inhibit the mixing (Lerche $\&$ Parker 1967). The entrained gas in that boundary layer might be able to propagate into the fossil following the field lines, but the large sizes of the fossils $\left(10 \ldots 10^{3} \mathrm{kpc}\right)$ would forbid their disintegration due to the gas leakage within the considered time scale. The relativistic particles contained in the old radio plasma have much larger gyro-radii $\left(10^{-9} \mathrm{kpc}(E / \mathrm{GeV})(B / \mu \mathrm{G})^{-1}\right)$ than the external gas particles. Scattering at magnetic irregularities allows the gyro-center of the particle motion to be displaced, leading to cross field diffusion required for particle escape. But even if the combined effect of cross field diffusion and rapid parallel diffusion along disordered field lines is considered (anomalous diffusion), only very special turbulent conditions allow an efficient escape of relativistic particles from radio plasma (Enßlin 1999a). Strong turbulence itself is probably more destructive by shredding the magnetized plasma into smaller pieces. But it has been argued (Enßlin 1999a; Enßlin \& Kaiser 2000) that this should stop on scales where the energy density of the turbulent energy cascade is insufficient to overcome magnetic forces.

From purely an observational viewpoint there already exists good evidence that clouds of synchrotron plasma can survive and retain their identity within the ICM for at least up to $\sim 10^{8} \mathrm{yr}$, in the form of relic radio sources showing a relaxed structure but well defined boundaries. These fairly robust age estimates are based on interpreting the observed radio spectral steepening in terms of the inverse Compton losses of the relativisitic electrons against the ubiquitous cosmic microwave background photons (e.g., Komissarov \& Gubanov 1994; Venturi et al. 1998; Slee \& Roy 1998).

Finally, we note that other energy loss mechanisms of the electrons, as Coulomb and Bremsstrahlung losses, are inefficient at the considered energies $(1-10 \mathrm{GeV})$, even if a dense gas component were to exist inside the radio plasma (Enßlin \& Kaiser 2000). If indeed such a cold gas component were present inside the radio lobe, Parker-type instabilities (Parker 1966) might occur, helping to shred the lobe into smaller pieces. Also Rayleigh-Taylor-type instabilities of the surface of the light radio plasma might occur, if not supressed by the tension of the magnetic field lines. But it is questionable if these instabilities allow a microscopic mixing of the environmental gas with the radio plasma, since both phases are still separated by magnetic fields. Further, there is observational evidence that the density of entrained gas cannot be high in radio lobes and in cluster radio relics. Many lobes and relics exhibit radio polarization, which has sometimes values close to the upper limit allowed by synchrotron theory (Harris et al. 1993; Saripalli et al. 1996, e.g.). No intrinsic Faraday rotation, or intrinsic Faraday depolarization is detected in these systems. In the cases of detected depolarization it seems to be consisten with beam depolarization, or the Laing-Garrington effect (Garrington et al. 1988), which is external to the source. This argues against a dense thermal plasma component. Nevertheless, we discuss briefly in Sect. 7 some possible implications of strong microscopic mixing for the proposed model.

\section{The formalism}

The dimensionless momentum of an ultra-relativistic electron $p=P_{\mathrm{e}} /\left(m_{\mathrm{e}} c\right)$ within the radio plasma changes due to synchrotron losses proportional to the magnetic energy density $u_{\mathrm{B}}$, inverse Compton (IC) losses proportional to the cosmic microwave background radiation field energy density $u_{\mathrm{C}}$, and adiabatic losses or gains connected to the change of the volume $V$ of the radio plasma:

$-\frac{\mathrm{d} p}{\mathrm{~d} t}=a_{0}\left(u_{\mathrm{B}}+u_{\mathrm{C}}\right) p^{2}+\frac{1}{3} \frac{1}{V} \frac{\mathrm{d} V}{\mathrm{~d} t} p$

where $a_{0}=\frac{4}{3} \sigma_{\mathrm{T}} /\left(m_{\mathrm{e}} c\right)$. We do not consider bremsstrahlung and Coulomb losses in view of the very low particle density within the radio plasma. We further assume sufficient pitch angle scattering to keep the electron pitch angle distribution isotropic (Jaffe \& Perola 1973). After introduction of the compression ratio

$C(t)=V_{0} / V(t)$

and a temporary change to the variable $\tilde{p}(t)=$ $C(t)^{-1 / 3} p(t)$ Eq. (1) is easily integrable, yielding finally

$p\left(p_{0}, t\right)=\frac{p_{0}}{C(t)^{-\frac{1}{3}}+p_{0} / p_{*}(t)}$.

Here, we defined the characteristic momentum $p_{*}(t)$, which is given by

$\frac{1}{p_{*}(t)}=a_{0} \int_{t_{0}}^{t} \mathrm{~d} t^{\prime}\left(u_{\mathrm{B}}\left(t^{\prime}\right)+u_{\mathrm{C}}\left(t^{\prime}\right)\right)\left(\frac{C\left(t^{\prime}\right)}{C(t)}\right)^{\frac{1}{3}}$.

If the change in volume can be approximated to be a power-law in time,

$V(t)=V_{0}\left(\frac{t}{t_{0}}\right)^{b}$, or $C(t)=\left(\frac{t}{t_{0}}\right)^{-b}$,

which we will assume in the following, an analytic solution to Eq. (1) was already given in Kaiser et al. (1997). We assume further the photon energy density to be constant, which is reasonable in our application since it is dominated by the cosmic microwave background, which does not change significantly on the time-scales considered here. Further, we assume that the magnetic field energy density scales as

$u_{\mathrm{B}}(t)=u_{\mathrm{B}, 0}\left(V / V_{0}\right)^{-4 / 3}=u_{\mathrm{B}, 0}\left(t / t_{0}\right)^{-4 b / 3}$,

as one would expects for an isotropic expansion of the magnetized plasma. These assumptions allow one to evaluate the integral in Eq. (4). We exclude the cases $b=3$ and $b=3 / 5$ for simplicity only (they produce logarithms 
instead of power-law relations), and write the characteristic electron momentum in a compact form:

$p_{*}(t)=\frac{C^{\frac{1}{3}}}{a_{0} t\left(\frac{C^{5 / 3}-C^{1 / b}}{1-5 b / 3} u_{\mathrm{B} 0}+\frac{C^{1 / 3}-C^{1 / b}}{1-b / 3} u_{\mathrm{C}}\right)}$

( $C=C(t)$ for brevity). It is obvious that the synchrotronand IC- cooling produces a sharp upper cutoff in the electron distribution $f(p, t)$ at $p_{*}(t)$ even if the original distribution $f_{0}\left(p_{0}\right)$ at time $t_{0}$ extended to infinity. The electron density per volume and momentum $f(p, t) \mathrm{d} p \mathrm{~d} V$ for $p<p_{*}(t)$ is given by

$f(p, t)=f_{0}\left(p_{0}(p, t)\right) \frac{\partial p_{0}(p, t)}{\partial p} C(t)$,

where

$p_{0}(p, t)=\frac{p C(t)^{-\frac{1}{3}}}{1-p / p_{*}(t)}$.

If the original distribution function was a power-law

$f_{0}\left(p_{0}\right)=\tilde{f}_{0} p_{0}^{-\alpha_{\mathrm{e}}}$

for $p_{\min 0}<p_{0}<p_{\max 0}$ the resulting spectrum is

$f(p, t)=\tilde{f}_{0} C(t)^{\frac{\alpha_{\mathrm{e}}+2}{3}} p^{-\alpha_{\mathrm{e}}}\left(1-p / p_{*}(t)\right)^{\alpha_{\mathrm{e}}-2}$

for $p_{\min }(t)=p\left(p_{\min 0}, t\right)<p<p_{\max }(t)=p\left(p_{\max 0}, t\right)$.

We are interested in the situation where several phases of cooling characterized by different expansion or contraction rates and durations shaped the electron distribution. We write $p_{1}=p\left(t_{1}\right)$ for the momentum of an electron originally at $p_{0}$ after phase 1 , which is characterized by the compression during this phase $C_{01}=C\left(t_{1}\right)$ and $p_{* 01}=p_{*}\left(t_{1}\right), p_{2}$ for the momentum of the same electron after phase 2 , characterized by $C_{12}$ and $p_{* 12}$, and so on. It is straightforward to show that the final electron momentum $p_{n}$ after $n$ such phases can still be written in the form

$p_{n}\left(p_{0}\right)=\frac{p_{0}}{\left(C_{0 n}\right)^{-\frac{1}{3}}+p_{0} / p_{* 0 n}}$,

where

$C_{0 n}=\prod_{i=1}^{n} C_{i-1 i}=\prod_{i=1}^{n} \frac{V_{i}-1}{V_{i}}=\frac{V_{0}}{V_{n}}$

is the compression ratio between original and final configuration and maximal final momentum is given by

$\frac{1}{p_{* 0 n}}=\sum_{i=1}^{n} \frac{\left(C_{0 i-1}\right)^{\frac{1}{3}}}{p_{* i-1 i}}$

The effects of the individual cooling phases sum up weighted by $\left(C_{0} i\right)^{\frac{1}{3}}$. Thus, whenever the radio plasma is most extended, cooling is inefficient.

It remains to provide the parameters describing the different phases. Suppose we want to describe a phase $i$ where the expansion or compression is described by $b_{i}$, and two of the following three quantities are given: $\tau_{i}$, the time scale of expansion, $C_{i-1 i}$ the compression ratio during the phase, and $\Delta t_{i}$ the duration of the phase. Theses quantities are related via

$C_{i-1 i}=\left(1+\Delta t_{i} / \tau_{i}\right)^{-b_{i}}$.

We get

$p_{* i-1 i}=\frac{C^{\frac{1}{3}}}{a_{0} t_{i}\left(\frac{C^{5 / 3}-C^{1 / b}}{1-5 b / 3} u_{\mathrm{B} i-1}+\frac{C^{1 / 3}-C^{1 / b}}{1-b / 3} u_{\mathrm{C}}\right)}$

with $C=C_{i-1 i}$ and $t_{i}=\tau_{i}+\Delta t_{i}$ for brevity. The magnetic energy density at the beginning of phase $i$ is that of the end of phase phase $i-1$ :

$u_{\mathrm{B} i-1}=u_{\mathrm{B} 0}\left(C_{0 i-1}\right)^{4 / 3}$.

The resulting electron spectrum from an initial power-law distribution is

$f_{i}(p)=\tilde{f}_{0} C_{0 i}^{\frac{\alpha_{\mathrm{e}}+2}{{ }^{3}}} p^{-\alpha_{\mathrm{e}}}\left(1-p / p_{* 0} i\right)^{\alpha_{\mathrm{e}}-2}$,

for $p_{\min i}=p_{i}\left(p_{\min 0}\right)<p<p_{\max i}=p_{i}\left(p_{\max 0}\right)$ and $f_{i}(p)=0$ otherwise. The synchrotron emission at a given frequency $\nu$ is

$L_{\nu i}=c_{3} B_{i} V_{i} \int_{p_{\min i}}^{p_{\max i}} \mathrm{~d} p f_{i}(p) \tilde{F}\left(\nu / \nu_{i}(p)\right)$,

where $c_{3}=\sqrt{3} \mathrm{e}^{3} /\left(4 \pi m_{\mathrm{e}} c^{2}\right)$ and the characteristic frequency is $\nu_{i}(p)=3$ e $B_{i} p^{2} /\left(4 \pi m_{\mathrm{e}} c\right)$. The dimensionless spectral emissivity of a mono-energetic isotropic electron distribution in isotropically oriented magnetic fields $\tilde{F}(x)$ can be approximated (Enßlin et al. 1999):

$\tilde{F}(x) \approx \frac{2^{2 / 3}(\pi / 3)^{3 / 2}}{\Gamma(11 / 6)} x^{1 / 3} \exp \left(-\frac{11}{8} x^{7 / 8}\right)$.

In reality, after shock passage an originally isotropic ensemble of field lines gets partially aligned with the shock plane. This is also true for the unshocked radio plasma, since its morphology gets significantly flattened during compression (see phase 3 in Sect. 5). This would produce a radio polarization and a luminosity which depends on the viewing angle (Enßlin et al. 1998). As long as we are only calculating the total luminosity of the radio cocoon/relic, this can be ignored. But, in case one wants to know the expected flux, one has to correct for the anisotropic emission pattern of the cluster radio relics. Fortunately, the degree of radio polarization can be used to determine the viewing angle with respect to the shock plane (Laing 1980; Enßlin et al. 1998).

The upper cutoff in the electron distribution at $p_{* 0} n$ produces a cutoff in the synchrotron spectrum near $\nu_{* n}=$ $\nu_{\mathrm{c}}\left(p_{* 0 n}\right)$. But since $\tilde{F}(x)$ has a broad maximum even a sharp cutoff in the electron spectrum gives a soft cutoff in the radio, with significant flux above $\nu_{* n}$. 
Table 1. Parameters of the three scenarios. The rows labled with "Phase 0" give the initial parameters of the radio plasma at the end of the activity of the radio galaxy. The rows below ("Phases 1-4") give the subsequent evolution, due to aging and expansion or compression described by the left block of parameters. Details can be found in the text. The luminosity units allow an easy conversion to the observable flux: for a source in $100 \mathrm{Mpc}$ distance the flux of 1 Jansky corresponds to a luminosity of $1.210^{31} \mathrm{erg} \mathrm{s}^{-1} \mathrm{~Hz}^{-1}$

\begin{tabular}{|c|c|c|c|c|c|c|c|c|c|c|c|c|}
\hline & $\begin{array}{l}\Delta t_{i} \\
\text { Gyr }\end{array}$ & $\begin{array}{l}\tau_{i} \\
\text { Gyr }\end{array}$ & $C_{i-1 i}$ & $\overline{b_{i}}$ & $\overline{C_{0 i}}$ & $\begin{array}{l}V_{n} \\
\mathrm{Mpc}^{3}\end{array}$ & $\begin{array}{l}B_{i} \\
\mu \mathrm{G}\end{array}$ & $\begin{array}{l}u_{\mathrm{B} i} \\
\mathrm{eV} \mathrm{cm}\end{array}$ & $p_{* 0 i}$ & $\begin{array}{l}\nu_{* i} \\
\mathrm{GHz}\end{array}$ & \multicolumn{2}{|c|}{$\begin{array}{c}L_{0.1 \mathrm{GHz}} \quad L_{1 \mathrm{GHz}} \\
10^{31} \mathrm{erg} \mathrm{s}^{-1} \mathrm{~Hz}^{-1}\end{array}$} \\
\hline $\begin{array}{l}\text { Scenario A } \\
\text { Phase } 0\end{array}$ & 0 & 0.015 & 1. & 1.8 & 1. & 0.0059 & 12. & 3.6 & $\infty$ & $\infty$ & 530. & 93. \\
\hline Phase 1 & 0.0054 & 0.01 & 0.595 & 1.2 & 0.595 & 0.0099 & 8.5 & 1.8 & 36700 . & 48. & 210. & 33. \\
\hline Phase 2 & 0.1 & $\infty$ & 1. & 0 & 0.595 & 0.0099 & 8.5 & 1.8 & 2730 . & 0.27 & 86. & 0.22 \\
\hline Phase 3 & 0.069 & -0.11 & 6.45 & 2. & 3.83 & 0.0015 & 29. & 22. & 1220. & 0.19 & 1500. & 0.94 \\
\hline Phase 4 & 0.02 & $\infty$ & 1. & 0 & 3.83 & 0.0015 & 29. & 22. & 651. & 0.052 & 240. & 0 \\
\hline Scenario B & & & & & & & & & & & & \\
\hline Phase 0 & 0 & 0.015 & 1. & 1.8 & 1. & 0.12 & 2.7 & 0.18 & $\infty$ & $\infty$ & 38. & 6.7 \\
\hline Phase 1 & 0.17 & 0.01 & 0.0316 & 1.2 & 0.0316 & 3.7 & 0.27 & 0.0018 & 8810. & 0.088 & 0.0096 & 0 \\
\hline Phase 2 & 1. & $\infty$ & 1. & 0 & 0.0316 & 3.7 & 0.27 & 0.0018 & 1830. & 0.0038 & 0 & 0 \\
\hline Phase 3 & 0.55 & -0.67 & 31.6 & 2. & 1. & 0.12 & 2.7 & 0.18 & 3270. & 0.12 & 7.4 & 0.00035 \\
\hline Phase 4 & 0.3 & $\infty$ & 1. & 0 & 1. & 0.12 & 2.7 & 0.18 & 1910. & 0.041 & 0.98 & 0 \\
\hline $\begin{array}{l}\text { Scenario C } \\
\text { Phase } 0\end{array}$ & 0 & 0.015 & 1. & 1.8 & 1. & 0.0059 & 1.2 & 0.03 & $\infty$ & $\infty$ & 0.3 & 0.053 \\
\hline Phase 1 & 0.032 & 0.01 & 0.178 & 1.2 & 0.178 & 0.033 & 0.38 & 0.0036 & 54800 . & 4.8 & 0.014 & 0.00099 \\
\hline Phase 2 & 0.1 & $\infty$ & 1. & 0 & 0.178 & 0.033 & 0.38 & 0.0036 & 16200. & 0.42 & 0.0078 & 0.000028 \\
\hline Phase 3 & 0.13 & -0.17 & 15.9 & 2. & 2.83 & 0.0021 & 2.4 & 0.14 & 16500. & 2.7 & 1.4 & 0.12 \\
\hline Phase 4 & 0.1 & $\infty$ & 1. & 0 & 2.83 & 0.0021 & 2.4 & 0.14 & 7860 & 0.62 & 1. & 0.02 \\
\hline
\end{tabular}

\section{The model}

Between the release from a radio galaxy and the reappearance as a cluster radio relic the radio plasmon undergoes several different phases of expansion and contraction:

Phase 0: Injection. The radio galaxy is active and a large expanding volume is being filled with radio plasma. The expansion of this cocoon is likely to be supersonic (with respect to the outer medium) and therefore $b_{0}=9 / 5$, if we assume that there is no gas density gradient in the vicinity of the radio galaxy (Kaiser \& Alexander 1997). We assume that the injection occurred with a time constant of $\tau=0.15$ Gyr, which is the typical age of a radio source at the end of nuclear activity (Alexander \& Leahy 1987, for typical ages). The particle population is kept close to a power-law distribution by injection of fresh electrons. We assume a spectral index of $\alpha_{\mathrm{e}}=2.5$ and an upper cutoff at $p_{\max 0}=10^{5}$. The momentum cut-off produces a radio cut-off above $42 \mathrm{GHz}(B / \mu \mathrm{G})$, but we note that our results are not very sensitive to the choice of this parameter. More realistic electron spectra at the end of phase 0 could be constructed by superimposing the spectra of the electron populations of different ages, as in the models of Kaiser et al. (1997). But, for the present purpose of demonstrating that the fossil radio plasma can be revived by compression, our simplified treatment should be sufficiently illustrative.

Phase 1: Expansion. After the central engine of the radio galaxy became inactive, the radio cocoon might still be strongly over-pressured compared to its gaseous environment (Begelman \& Cioffi 1989). If this is the case a Sedov-like expansion phase exists with $b_{1}=6 / 5$.
Momentum conservation of the expanding shell around the cocoon forces the expansion rates at the end of phase 0 and beginning of phase 1 to be the same, leading to $\tau_{1}=b_{1} / b_{0} \tau_{0}=2 / 3 \tau_{0}$. The expansion will significantly deviate from $b_{1}=6 / 5$ at the moment when the internal pressure drops to a value comparable to the environmental pressure. We simplify this behavior by assuming that the expansion is Sedov-like until pressure equilibrium is reached. The radio cocoon probably becomes undetectable during this phase, and therefore becomes a fossil radio cocoon or a so called radio ghost.

Phase 2: Lurking. Pressure equilibrium with the environment is reached, and the volume of the radio plasma remains more or less constant. Thus $C_{12} \approx 1$ and $b \approx 0$. Taking the appropriate limit $\tau_{2} \rightarrow \infty$ of Eq. (16) gives the well known result

$p_{* 12}=\left[a_{0}\left(u_{\mathrm{B} 2}+u_{\mathrm{C}}\right) \Delta t_{2}\right]^{-1}$.

Due to the previous adiabatic energy losses of the electrons, they reside at low energies during phase 2. Their radiation losses, which strongly depend on the particle energies, are therefore strongly diminished. Additionally, the synchrotron losses are further reduced due to the weaker magnetic field during the expanded state of the radio cocoon. The adiabatic losses are reversible, and will be reversed during the subsequent compression phase, whereas the radiative losses are irreversible. Since the latter are suppressed during this phase, the radio ghost state can be called the energy saving mode of a radio cocoon.

Phase 3: Flashing. The fossil radio plasma gets dragged into a shock wave of cosmic large-scale structure formation, e.g. at the boundary of a cluster of galaxies or 
in a galaxy cluster merger event. While its thermal environment gets shocked, the radio plasma itself is only compressed adiabatically, due to the much higher internal sound speed. The electron population and the magnetic field gain energy adiabatically, leading to a steep enhancement of the synchrotron emissivity.

This phase has a duration of the order of $\Delta t_{3}=$ $V_{2}^{1 / 3} / v_{\text {shock } 2} \approx 0.1 \ldots 1 \mathrm{Mpc} /\left(300 \ldots 3000 \mathrm{~km} \mathrm{~s}^{-1}\right) \approx$ 30 Myr... 3 Gyr. The pre-shock flow velocity in the shock

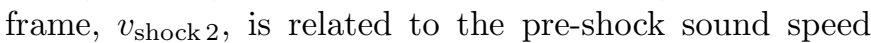
$c_{\mathrm{s} 2}=\sqrt{\gamma P_{2} /\left(n_{\mathrm{e}}\left(m_{\mathrm{p}}+m_{\mathrm{e}}\right)\right)}$ via

$v_{\text {shock } 2}^{2}=\frac{c_{\mathrm{s} 2}^{2}}{2 \gamma}\left(\gamma-1+(\gamma+1) \frac{P_{3}}{P_{2}}\right)$

(Landau \& Lifschitz 1966), where $\gamma=5 / 3$ is the adiabatic index of the thermal gas. The compression factor of the relativistic plasma is high, and can be calculated from the assumed pressure jump $P_{3} / P_{2}$ of the surrounding thermal gas, assuming pressure equilibrium before and after the shock passage:

$C_{23}=\left(P_{3} / P_{2}\right)^{\frac{3}{4}}$

In order to guess the parameters $b_{3}$ and $\tau_{3}$ a rough picture of the compression process is required. The sound speed within the radio plasma should be much higher than even in the post-shock thermal environment, (it could be up to $c / \sqrt{3}$, if the plasma is fully relativistic), so that an instantaneous response to environmental changes can be assumed. During the shock passage, the cocoon is exposed to the high thermal pressure of the post-shock gas on its down- and to the ram-pressure of the pre-shock gas on its up-stream side. But, on the remaining surface the cocoon is only subject to the (much lower) up-stream gas pressure. The relativistic plasma will therefore start to expand orthogonal to the gas flow, producing a flattened pancake-like morphology. In order be able to expel the ambient gas sideways additional internal pressure comparable to the ram pressure of the expelled material is needed. This pressure is produced by compression. The process of flattening stops when the ram pressure of the swept-up material at the expanding edges of the pancake is of the order of the ram pressure of the incoming flow. This implies that the ratio of the diameter to the thickness of the expanded cocoon is roughly 4 for a strong shock.

The compression is slow in the beginning, and rapid towards the end when the cocoon is significantly flattened. We mimic this by setting $b_{3}=2$ and a negative $\tau_{3}$, according to Eq. (15). We favor this over the more intuitive choice of a positive $\tau_{3}$ and large negative $b_{3}$, since it describes the process of compression more realistically.

Phase 4: Fading. The radio plasma is in pressure equilibrium with the post-shock medium, which should provide roughly a uniform environment: $b_{4}=0$. The radio emission of the relic now fades away due to the heavy radiation losses.

\section{The scenarios}

We follow the evolution of a cocoon of radio plasma for three different scenarios (A, B, and $\mathrm{C}$ ) and calculate the resulting radio emission. These scenarios are envisioned to illustrate plausible situations, rather than try to reproduce precise parameters for some known cluster radio relics. Scenario A and B are chosen to represent extreme cases: in $\mathrm{A}$, the relic is located at the center of a galaxy cluster, while in B, its location is near the cluster boundary, i.e., in the proximity of the accretion shock wave. In both scenarios the duration of phase 2 was chosen to be so long that the shocked radio plasma could be barely observed as a weak ultra-steep spectrum source. Since these scenarios are rather extreme, we demonstrate with scenario $\mathrm{C}$ that a shorter phase 2 can result in a moderately steepened spectrum of the cluster radio relic.

For the cases A and B, we assume the initial cocoon (at the end of phase 0 ) to contain magnetic fields, relativistic electrons and protons with energies of $E_{\mathrm{B} / \mathrm{e} / \mathrm{p}}=10^{60} \mathrm{erg}$ each, and $E_{\mathrm{B} / \mathrm{e} / \mathrm{p}}=10^{58} \mathrm{erg}$ in case C. The three components produce a relativistic isotropic pressure of

$P_{\text {cocoon } 0}=\frac{E_{\mathrm{e}}+E_{\mathrm{p}}+E_{\mathrm{B}}}{3 V_{0}}$.

We assume a spectral index of $\alpha_{\mathrm{e}}=2.5$ and a rather high cutoff in the electron spectrum at $p_{\max 0}=10^{5}$, which has no significant influence on the conclusions. The lower cutoff is $p_{\min 0}=10$ in scenarios $\mathrm{A}$ and $\mathrm{B}$, and $p_{\min 0}=100$ in $\mathrm{C}$. The lower cutoffs only affect the normalizations of the radio fluxes, not the spectral shapes.

The different scenarios are described below in detail. The parameters for the different scenarios are listed in Table 1 and the resulting radio spectra at the end of the different phases are shown in Figs. 2-4.

Scenario A: The cocoon at the cluster center. The pre-merger cluster is assumed to have an electron density of $n_{\mathrm{e}}=0.310^{-3} \mathrm{~cm}^{-3}$ and a temperature of $k T=3 \mathrm{keV}$ at the location of the radio galaxy. Due to the high environmental pressure, we assume the internal pressure of the cocoon to be only twice the external pressure, and use Eq. (24) to obtain the initial volume $V_{0}$. Due to the strong synchrotron energy losses in this scenario, phase 2 can not last much longer than $\Delta t_{2}=0.1 \mathrm{Gyr}$, otherwise the revived fossil radio cocoon would not emit within the observable radio frequency range. We assume that the shock wave of a cluster merger event increases the internal pressure by $P_{3} / P_{2}=12$ during the phase 3 . This corresponds to a moderate shock with shock compression factor of 2.8 , whereas strong non-relativistic shocks can have a compression factor of 4. A moderate shock is expected, since both merging clusters are expected to have temperatures of several $\mathrm{keV}$ and therefore sound velocities comparable to the merger velocity.

As can be seen in Fig. 2 the compression caused by the merger shock wave gives rise to a burst of low frequency emission, but practically no high frequency emission. This is due to the rapid decay of the upper end of the electron spectrum during phase 3 , which essentially wipes out 


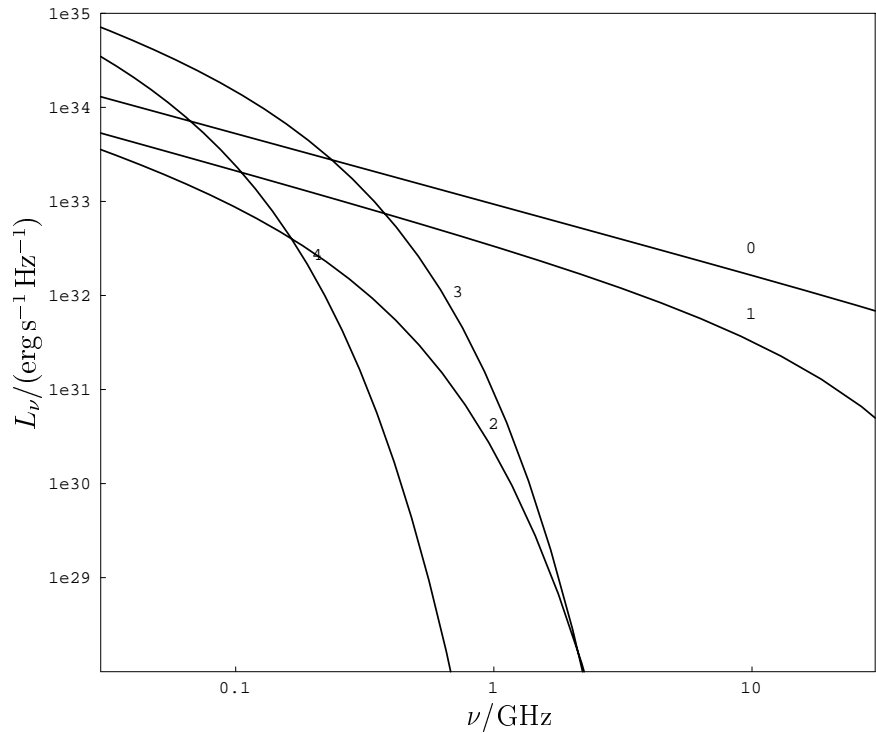

Fig. 2. Radio spectrum of the radio cocoon in scenario A at the end of phases $0-4$

the adiabatic energy gains of these electrons. The source decays on a time-scale of a few tens of Myr, mostly due to the heavy synchrotron losses. If the radio cocoon is located in a more peripheral region of the cluster, where the density, the pressure and therefore the magnetic field strength inside the cocoon is much lower, these losses are also much milder. This lengthens the time scale over which the radiatively cooling synchrotron plasma can still be revived by the next passing shock, and thus rendered radio detectable. We, therefore, expect the radio relic phenomena to be found preferentially at larger cluster radii, and less often near the cluster center (although projection can help some relics to appear near the cluster core). The best environment to find cluster radio relics is, therefore, near the edges of the clusters.

Scenario B: The cocoon at the cluster boundary. The radio cocoon is assumed here to be born outside the cluster, in an environment of a dense galaxy filament, or a group of galaxies, say, with $n_{\mathrm{e}}=0.310^{-5} \mathrm{~cm}^{-3}$ and $k T=0.3 \mathrm{keV}$. The freshly injected radio plasma might be over-pressured by a factor of 100 , leading to a short expansion phase. After this, the electrons within the expanded Mpc sized cocoon suffer mostly the IC-losses, allowing revival of the radio plasma even $\Delta t_{2}=1$ Gyr later. This can happen when the cocoon along with the ambient medium is crossed by the accretion shock of a cluster of galaxies, which might entail a pressure jump as large as $P_{3} / P_{2}=100$, in order to heat the infalling cool gas to the cluster virial temperature of up to $10 \mathrm{keV}$.

Scenario B can explain the steep and bent radio spectrum of the cluster radio relic 0038-096 in Abell 85 . An eye-fit to the radio spectrum (Fig. 5) shows that the maximal electron momentum in this case is $p_{*}=$ $10^{4}(B / \mu \mathrm{G})^{-1 / 2}$. The magnetic field strength of the cluster relic was estimated from the minimum energy argument to be $B \approx 1 \mu \mathrm{G}$ (Feretti \& Giovannini 1996)

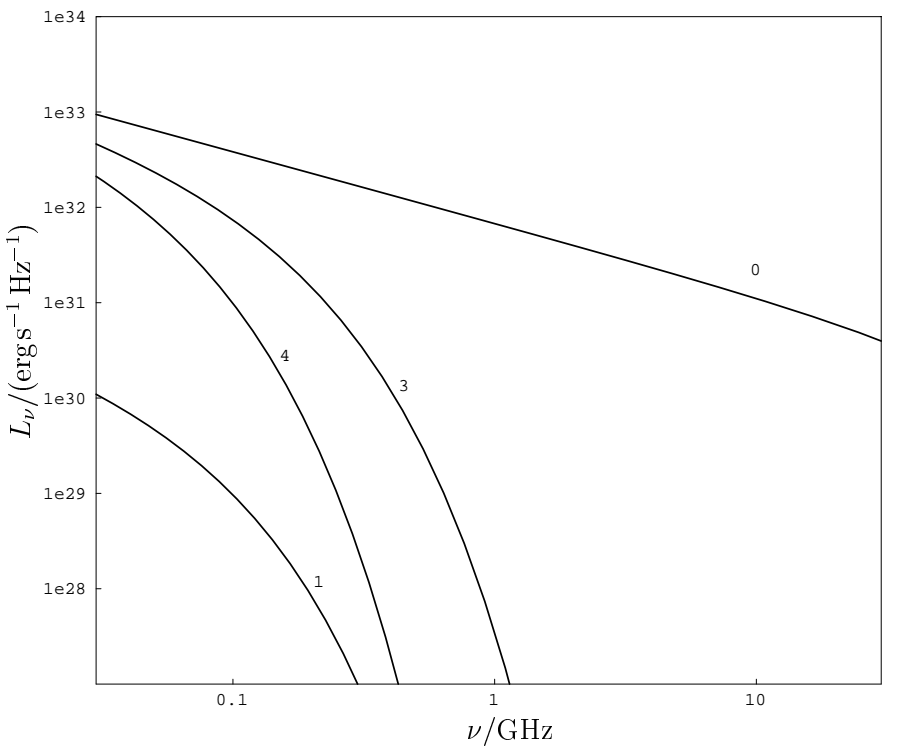

Fig. 3. Radio spectrum of the radio cocoon in scenario B at the end of phases $0-4$. The luminosity at the end of phase 2 is too small in order to be displayed in this figure

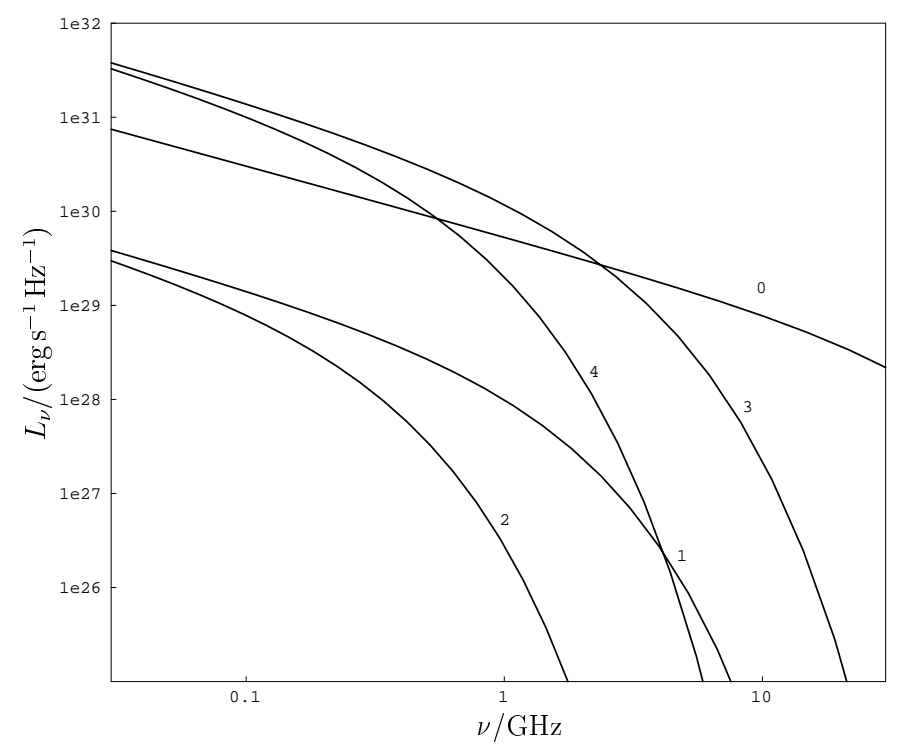

Fig. 4. Radio spectrum of the radio cocoon in scenario $\mathrm{C}$ at the end of phases $0-4$

and from the detection of excess X-ray emission at the location of the relic, which implies a field strength of $B=0.95 \pm 0.10 \mu \mathrm{G}$ (Bagchi et al. 1998) if this emission refers to the IC scattered cosmic microwave background photons, otherwise a higher field strength. Using $B=1 \mu \mathrm{G}$ and $p_{*}=10^{4}$ and assuming a uniform environment without expansion and compression, an age of 0.2 Gyr would result (Komissarov \& Gubanov 1994, and see Eq. (21)). But scenario B demonstrates that the radio plasma can be as old as 2 Gyr. This resolves the problem of the apparent cooling time of the electrons being too short for any nearby galaxy to have ejected the plasma and then moved to its present location with a typical velocity of a cluster 


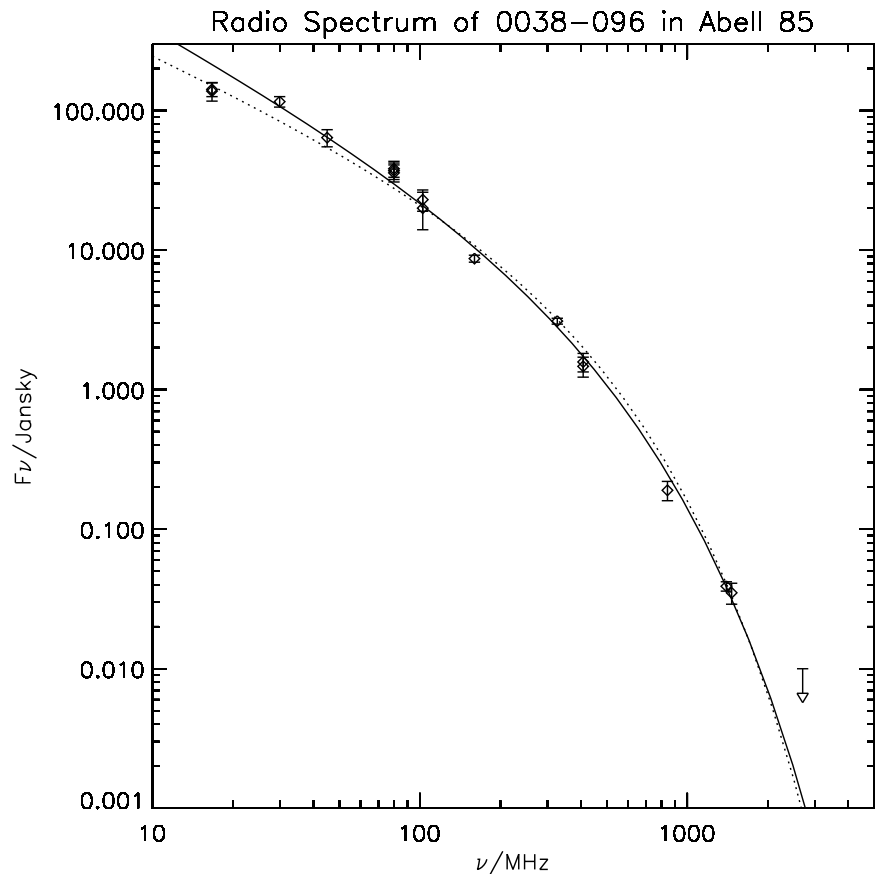

Fig. 5. Radio spectrum of the radio relic in A85. The data was compiled from the literature by Bagchi et al. (1998). The solid line is a radio spectrum resulting from the canonical form (Eq. (18)) with $\alpha_{\mathrm{e}}=3$ and $p_{*}=11000(B / \mu \mathrm{G})^{-1 / 2}$, and the dotted line one with $\alpha_{\mathrm{e}}=2.65$ and $p_{*}=10000(B / \mu \mathrm{G})^{-1 / 2}$

member. For the long duration of phase 2 the resulting spectrum is fairly steep in the observable radio range. But this need not to be the case for a scenario with a shorter fossil phase.

Scenario C: The smoking gun. In order to substantiate the last statement, we choose a set of parameters for scenario $\mathrm{C}$ which produces a cluster radio relic with relative flat, nearly unbent radio spectrum, like the relic $1253+275$ at the boundary of the Coma cluster of galaxies (Giovannini et al. 1991, and see Fig. 1). There, the probable source of the radio plasma is visible: it is the galaxy NGC 4789, which is located upstream of the relic, and which seems to be on an ascending orbit after a cluster core passage (Enßlin et al. 1998). Its narrow angle tailed radio outflow - the smoke of the gun NGC 4789 - seems to be bent and dragged by the infalling matter (falling into Coma's gravitational potential) to the location of the relic. There, it brightens up and exhibits a steep but straight spectrum with a slope of 1.18. For details of the spectrum and the geometry, see Giovannini et al. (1991) and Enßlin et al. (1998).

We follow a blob of the radio plasma, which might have been injected with an overpressure of a factor 10 into the infalling gas stream with possibly $n_{\mathrm{e}}=0.310^{-5} \mathrm{~cm}^{-3}$ and $k T=0.6 \mathrm{keV}$. The inflow of the plasma and the compression at the cluster accretion shock wave might have taken a few hundreds of Myr. We assume a pressure jump of only $P_{3} / P_{2}=40$ at the shock wave, not higher, in order to allow the temperature of the post-shock gas to stay below the average cluster temperature of $8.2 \mathrm{keV}$
(Briel et al. 1992). As can be seen in Fig. 4, the radio spectrum below $1 \mathrm{GHz}$ stays practically unbent for a couple of tens of Myr after the shock passage.

\section{Discussion}

We have argued here that adiabatic compression in cluster shocks can revive fossil radio plasma to radio detection, even up to 2 Gyr after its release from the parent radio galaxy. The computed radio spectra provide a good match to the observed spectra of cluster radio relics. Hence, this is a promising model for the regions of diffuse radio emission found in clusters of galaxies. Below, we summarize some merits and potential shortcoming of this model:

\section{Pros:}

- The observed connection of cluster radio relics to shock waves arises naturally in this model;

- The presence of the radio galaxy NGC 4789 and the morphological connection of its radio tails to the relic $1253+275$ seems to be the smoking gun of cluster radio relic formation by compression of fossil radio plasma;

- The expected very high sound speed within radio plasma should virtually forbid shock waves of the ambient medium to penetrate into radio cocoons. This renders adiabatic compression as a more plausible means of reviving the fossil plasma;

- Cluster radio relics are rare. Not only a shock wave and fossil radio plasma have to be present in this model, in order to produce a cluster radio relic, but also the fossil radio plasma can not grow too old (of the order of 0.1 Gyr in the center of clusters and 1 Gyr at their boundaries). Otherwise the high energy end of the electron population would suffer too severe a depletion for the adiabatic gains during the compression to be able to shift them to radio emitting energies. Since fossil radio plasma should be very common (Enßlin \& Kaiser 2000 ), this helps to explain the observed rarity of cluster radio relics;

- The observed tendency of relics to appear at peripheral positions in clusters follows in this model from the much shorter lifetimes of the radio emission and, consequently, much shorter ages which the revive-able fossil radio cocoons can have in the center of clusters, owing to the stronger synchrotron losses occurring there.

\section{Contras:}

- If the radio plasma has a high mass load, due to undetectable cool gas, it should get shocked, ensuing Fermi-I shock acceleration to produce the observed radio emitting electron population (Enßlin et al. 1998). Still, the adiabatic gains of the relativistic particles are inescapable, although restricted in this case due to the limited compression factor of a shock wave;

- In the case when the fossil radio plasma is supported by a very hot, non-relativistic gas component, both adiabatic and shock acceleration are ineffective. The first 
due to the lower compressibility of a non-relativistic equation of state, while the second due to the inability of environmental shock waves to penetrate into the plasma. The fact that we do observe cluster radio relics tells us that this situation does not necessarily arise (or, alternatively, cluster radio relics are not related to radio ghosts);

- The giant cluster radio relic 2006-56 in Abell 3667 shows a rim of flat radio emission (spectral index around 0.5) at its sharp outer edge (Röttgering et al. 1997). This could be understood in a Fermi-I shock acceleration model due to the different ages of the electrons found at different distances from the energizing shock wave (Enßlin et al. 1998). There are two possibilities to explain the observation of a flat spectrum rim within the adiabatic compression model: (a) there is a high mass load in the radio plasma, allowing the shock wave to successively compress the plasma, which should also produce a spatially different electron population, and (b) the apparent rim of a flat spectral index is an artifact arising from an unmatched coverage of the Fourier space in the radio interferometric observations at the two frequencies used.

We conclude that the model presented here provides a fairly natural and promising explanation for the phenomenon of cluster radio relics. This model predicts the existence of a population of diffuse, ultra-steep, very low frequency radio sources inside and possibly also outside of clusters of galaxies, due to the age dependence of the upper frequency cutoff of radio emission arising from the revivable fossil radio plasma. The ongoing rapid improvements in the sensitivities at low radio frequencies (Giant Meterwave Radio Telescope: (GMRT), Low Frequency Array (LOFAR)) may therefore open a new window on cosmological structure formation by detecting shock waves marked by such relic radio sources.

Acknowledgements. We would like to thank Christian Kaiser and Peter Biermann for discussions and comments on the manuscript. We also thank Joydeep Bagchi, Vincent Pislar and Gastao Lima Neto for the compiled radio spectrum of 0038-096 and Gabriele Giovannini, Luigina Feretti, and Carlo Stanghellini for the radio data of $1253+275$. We are grateful to the anonymous referee for stimulating comments. TAE acknowledges the friendly hospitality at the National Center for Radio Astrophysics (NCRA) in Pune, where this work was initiated. He further thanks the DFG and the SOC of the IAU 199 conference for additional travel support.

\section{References}

Alexander, P., \& Leahy, J. P. 1987, MNRAS, 225, 1

Bagchi, J., Pislar, V., \& Lima Neto, G. B. 1998, MNRAS, 296, L23

Begelman, M. C., \& Cioffi, D. F. 1989, ApJL, 345, L21

Briel, U. G., \& Henry, J. P. 1994, Nature, 372, 439

Briel, U. G., Henry, J. P., \& Boehringer, H. 1992, A\&A, 259, L31

Churazov, E., Brüggen, M., Kaiser, C. R., Böhringer, H., \& Forman, W. 2000, ApJ, submitted [astro-ph/0008215]
Donnelly, R. H., Markevitch, M., Forman, W., et al. 1998, ApJ, 500,138

Enßlin, T. A. 1999a, in Ringberg Workshop on Diffuse Thermal and Relativistic Plasma in Galaxy Clusters, ed. P. S. H. Böhringer, L. Feretti, vol. 271 of MPE Report, 275 [astro-ph/9906212]

Enßlin, T. A. 1999b, in IAU Symp. 199, The Universe at Low Radio Frequencies [astro-ph/0001433]

Enßlin, T. A., Biermann, P. L., Klein, U., \& Kohle, S. 1998, A\&A, 332, 395

Enßlin, T. A., \& Kaiser, C. R. 2000, A\&A, 360, 417

Enßlin, T. A., Lieu, R., \& Biermann, P. L. 1999, A\&A, 344, 409

Feretti, L. 1999, in IAU Symp. 199, The Universe at Low Radio Frequencies [astro-ph/0006379]

Feretti, L., \& Giovannini, G. 1996, in IAU Symp. 175, Extragalactic Radio Sources, 175, 333

Garrington, S. T., Leahy, J. P., Conway, R. G., \& Laing, R. A. 1988, Nature, 331, 147

Giovannini, G., Feretti, L., \& Stanghellini, C. 1991, A\&A, 252,528

Giovannini, G., Tordi, M., \& Feretti, L. 1999, New Astron., 4,141

Harris, D. E., Stern, C. P., Willis, A. G., \& Dewdney, P. E. 1993, AJ, 105, 769

Jaffe, W. 1992, in Clusters and Superclusters of Galaxies, 109

Jaffe, W. J., \& Perola, G. C. 1973, A\&A, 26, 423

Kaiser, C. R., \& Alexander, P. 1997, MNRAS, 286, 215

Kaiser, C. R., Dennett-Thorpe, J., \& Alexander, P. 1997, MNRAS, 292, 723

Kaiser, C. R., Schoenmakers, A. P., \& Röttgering, H. J. A. 2000, MNRAS, 315, 381

Kang, H., Rachen, J. P., \& Biermann, P. L. 1997, MNRAS, 286, 257

Kang, H., Ryu, D., \& Jones, T. W. 1996, ApJ, 456, 422

Komissarov, S. S., \& Gubanov, A. G. 1994, A\&A, 285, 27

Kulsrud, R. M., Cen, R., Ostriker, J. P., \& Ryu, D. 1997, ApJ, 480, 481

Laing, R. A. 1980, MNRAS, 193, 439

Landau, L., \& Lifschitz, E. 1966, Hydrodynamik (AkademieVerlag, Berlin)

Lerche, I., \& Parker, E. N. 1967, ApJ, 150, 731

Loeb, A., \& Waxman, E. 2000, Nature, 405, 156

Miniati, F., Ryu, D., Jones, T., \& Kang, H. 2000a, in Cosmic Evolution and Galaxy Formation: Structure, Interactions, and Feedback, ed. J. Franco, E. Terlevich, O. Lopez-Cruz, \& I. Aretxaga, ASP Conf. Ser. [astro-ph/0005445]

Miniati, F., Ryu, D., Kang, H., et al. 2000b, ApJ, 542, 608

Norman, C. A., Melrose, D. B., \& Achterberg, A. 1995, ApJ, 454,60

Parker, E. N. 1966, ApJ, 145, 811

Quilis, V., Ibanez, J. M. A., \& Saez, D. 1998, ApJ, 502, 518

Roettiger, K., Burns, J. O., \& Stone, J. M. 1999, ApJ, 518, 603

Röttgering, H. J. A., Wieringa, M. H., Hunstead, R. W., \& Ekers, R. D. 1997, MNRAS, 290, 577

Ryu, D., Kang, H., \& Biermann, P. L. 1998, A\&A, 335, 19

Saripalli, L., Mack, K., Klein, U., Strom, R., \& Singal, A. K. 1996, A\&A, 306, 708

Siemieniec, G., \& Ostrowski, M. 2000, A\&A, 355, 51

Slee, O. B., \& Roy, A. L. 1998, MNRAS, 297, L86

Tozzi, P., Scharf, C., \& Norman, C. 2000, ApJ, 542, 106

Venturi, T., Bardelli, S., Morganti, R., \& Hunstead, R. W. 1998, MNRAS, 298, 1113

White, S. D. M., Briel, U. G., \& Henry, J. P. 1993, MNRAS, $261, \mathrm{~L} 8$ 